\title{
Associations between Markers of Glucose and Insulin Function and Cognitive Function in Healthy African American Elders
}

\author{
Jeannine S. Skinner ${ }^{1 *}$, Amy Morgan ${ }^{2}$, Hector Hernandez-Saucedo ${ }^{3}$, An \\ Leverenz $^{5}$, Consuelo H. Wilkins ${ }^{1}$, Suzanne Craft $^{3}$ and Laura D. Baker ${ }^{3}$ \\ ${ }^{1}$ Meharry-Vanderbilt Alliance, Vanderbilt University Medical Center, Nashville, TN, USA \\ ${ }^{2}$ Geriatric Research Education and Clinical Center, Veterans Affairs Puget Sound Health Care System, Seattle, WA, USA \\ ${ }^{3}$ Department of Internal Medicine, and Neurology, Wake Forest University Health Sciences, Winston-Salem, NC, USA \\ ${ }^{4}$ Division of Gerontology, Geriatric Medicine, University of Washington School of Medicine, USA \\ ${ }^{5}$ Lou Ruvo Center for Brain Health, Cleveland Clinic Foundation, Cleveland, Ohio, USA
}

\begin{abstract}
Background: Glucose and insulin are important moderators of cognitive function. African Americans have poorer glycemic control across the glycemic spectrum and are at increased risk for type 2 diabetes and poor cognitive health. It is unclear which glucoregulatory markers predict cognitive function in this at-risk population. The purpose of this study was to examine the association between cognitive function and common markers of glucoregulation in nondiabetic African Americans elders.
\end{abstract}

Methods: Thirty-four, community-dwelling African Americans, aged 50-75 years completed cognitive testing and blood collection as part of a health screening assessment. Cognitive outcomes were composite scores derived from neuropsychological tests of executive function and verbal memory. Linear regression was used to examine relationships between cognitive composite scores and fasting blood levels of glucose, insulin, and hemoglobin A1C, with adjustments for age, education, body mass index, and antihypertensive medication use.

Results: Fasting plasma glucose was negatively associated with executive function $(\beta=-0.41, p=0.03)$. There was a trend of an association between fasting plasma glucose and verbal memory $(\beta=-0.34, p=0.06)$. Fasting insulin and hemoglobin A1c were not associated with cognitive function.

Conclusion: High non-diabetic fasting glucose levels were associated with poorer executive function and verbal memory. These results provide preliminary support for proactive glucose control in older African Americans even before glycemic criteria for type 2 diabetes are met. Our findings suggests that high-normal FPG levels may represent an early red-flag to signify increased risk of cognitive impairment or decline.

Keywords: Glucose; Type 2 diabetes; Memory; Executive function; African Americans

\section{Introduction}

Type 2 diabetes mellitus (T2DM) is a risk factor for cardiovascular diseases [1], cognitive impairment [2] and dementia [3]. Approximately $30 \%$ of adults aged 65 years and older have diabetes; and rates among African Americans are higher than that of many other ethnoracial groups [4]. Similar findings have been reported for rates of dementia among African Americans [5]. Burgeoning evidence shows high-normal glucose levels may be associated with poorer cognitive function [6] and increased risk for cognitive decline [7-8]. Such findings are particularly important for African Americans as this group may experience poorer glycemic control across the glycemic spectrum $[9,10]$.

Executive function and processing speed, cognitive processes mediated by prefrontal brain regions, are particularly vulnerable to glucose and insulin abnormalities [6]. These domains are also among the first to be affected by Alzheimer's disease (AD) [11,12]. Neuroimaging reports show higher fasting glucose is associated with reduced regional cerebral metabolic rate for glucose (rCMRglu) in regions that typically show hypometabolism in AD [13], a finding that replicates some of our earlier work in adults with mild metabolic dysfunction (prediabetes) [14]. Hippocampal and amydalar atrophy have also been reported in individuals with high-normal FPG levels [15]. Other indices of glucoregulatory function such as fasting insulin and glycosylated hemoglobin A1c (HbAlc) levels, have also been linked to cognitive function. Hyperinsulinemia, a proxy measure of insulin resistance (IR), predicts cognitive decline [16,17]. Elevated insulin levels may also accelerate $\mathrm{AD}$ neuropathological processes [18], potentially by disrupting clearance of amyloid- $\beta(A \beta)$ [19], the hallmark constituent of the AD pathology. Glycosylated hemoglobin $(\mathrm{HbAlc})$ is an estimate of long-term glucose control. Studies examining the association between HbAlc and cognitive function have yielded mixed findings [7-8,20]. Disparities in study findings may be influenced by restricted HbAlc variability in non-diabetic populations. Collectively, these findings accentuate the importance of glycemic control, even in the absence of T2DM, to reduce the risk of cognitive impairment and decline.

Given that African Americans are disproportionately affected by T2DM [4] and vulnerable to cognitive decline and dementia $[5,21]$, an investigation that characterizes the relationship between glucose, insulin, and cognitive function, prior to the onset of disease, may help to inform intervention priorities aimed at glycemic control and T2DM prevention rather than T2DM management in this vulnerable population. The current study examined cross-sectional associations between glucoregulatory markers and cognitive function in domains most vulnerable to age-related and disease-related changes in glycemic control (executive function and verbal memory).

*Corresponding author: Jeannine Skinner, $\mathrm{PhD}$, Meharry-Vanderbilt Alliance Vanderbilt University Medical Center 1005 Dr. D.B. Todd Jr, Boulevard Nashville, TN 37208, USA, Tel: 615-963-2834; E-mail: Jeannine.s.skinner@vanderbilt.edu

Received June 30, 2015; Accepted July 20, 2015; Published July 23, 2015

Citation: Skinner JS, Morgan A, Hernandez-Saucedo $H$, Hansen A, Corbet $S$ (2015) Associations between Markers of Glucose and Insulin Function and Cognitive Function in Healthy African American Elders. J Gerontol Geriat Res 4 232. doi:10.4172/2167-7182.1000232

Copyright: (c) 2015 Skinner JS, et al. This is an open-access article distributed under the terms of the Creative Commons Attribution License, which permits unrestricted use, distribution, and reproduction in any medium, provided the original author and source are credited. 


\section{Materials and Methods}

\section{Participants}

Thirty-four African Americans elders (aged 50-89) who completed health screening for participation in research studies investigating the association between insulin/glucose abnormalities and cognitive aging were included in this study. Recruitment was conducted through advertisements in newspapers, health presentations at local churches and community centers, and blood pressure checks at a predominately African American senior center. Participants were excluded from the study if they had major psychiatric or neurologic disorders, alcoholism, renal or hepatic disease, diabetes mellitus, chronic obstructive pulmonary disease, or unstable cardiac disease. Uses of antihypertensive and cholesterol-lowering medications were permitted while diabetes medications were not. Sample characteristics are provided in Table 1. The institutional review boards of the University of Washington and Veterans Affairs Puget Sound Health Care System approved this study.

Cognitive diagnosis (to rule out dementia) was determined by consensus by an expert panel of physicians and neuropsychologists with experience adjudicating such cases in geriatric populations. All available information from the screening visit was used to make this decision, including cognitive test results, medical and social history, physical examination, and clinical laboratory blood tests. Dementia was an exclusion, and all participants had hemoglobin A1C values $<6.5 \%$ (threshold for T2DM).

\section{Procedure}

Cognitive testing and 12-hr fasting blood collection occurred between $8 \mathrm{am}$ and $10 \mathrm{am}$. Measurements in blood included safety labs, glucose and insulin concentration, and HbA1C. Height, weight, and blood pressure measurements were recorded by study personnel.

\section{Cognitive measures}

Executive function (EF) was assessed using performance on Trails-B of the Trail-Making Test [22], Digit-Symbol [23], Digit Span Backwards [23], and Verbal Fluency (letter and category) [24]. Verbal

\begin{tabular}{|l|c|c|c|}
\hline & Females & Males & Total \\
\hline N & 27 & 7 & 34 \\
\hline Age, years & $60.9(6.5)$ & $57.5(7.6)$ & $60.2(6.7)$ \\
\hline Education, years & $15.8(2.3)$ & $14.7(2.8)$ & $15.2(2.1)$ \\
\hline BMI, $\mathrm{m}^{2} / \mathrm{kg}$ & $33.8(6.0)$ & $30.0(2.5)$ & $33.1(5.6)$ \\
\hline Fasting glucose, $\mathrm{mg} / \mathrm{dL}$ & $94.4(9.9)$ & $101.4(4.5)$ & $95.8(9.4)$ \\
\hline Fasting insulin, $\square \mathrm{U} / \mathrm{mL}$ & $11.5(6.3)$ & $12.8(4.9)$ & $11.7(6.1)$ \\
\hline Hemoglobin A1C, \% & $5.7(.29)$ & $5.7(.19)$ & $5.7(.27)$ \\
\hline 3MSE (max=100) & $95.8(4.2)$ & $93.2(3.4)$ & $95.2(4.1)$ \\
\hline Executive Function & & & \\
\hline TMT-B, sec & $82.6(30.0)$ & $92.0(50.0)$ & $84.5(34.3)$ \\
\hline Digit Span Backwards & $4.6(1.4)$ & $4.5(1.1)$ & $4.6(1.3)$ \\
\hline Digit-Symbol & $51.5(17.3)$ & $46.8(15.2)$ & $50.4(16.8)$ \\
\hline Word Fluency & $34.0(7.7)$ & $33.1(3.9)$ & $33.8(7.0)$ \\
\hline Verbal Memory & & & \\
\hline CVLT - immediate free recall & $9.7(2.6)$ & $7.8(4.3)$ & $9.3(3.1)$ \\
\hline CVLT - delayed free recall & $10.4(2.4)$ & $7.2(4.5)$ & $9.7(3.1)$ \\
\hline Logical Memory - immediate & $25.4(6.6)$ & $21.5(6.9)$ & $24.6(6.7)$ \\
\hline Logical Memory - delayed & $25.8(7.2)$ & $19.8(7.4)$ & $24.6(7.5)$ \\
\hline
\end{tabular}

Mean (SD); Abbreviations: BMI: Body Mass Index; 3MSE: Modified Mini-Mental Status Examination; TMT-B: Trail Making Test, part B; Word Fluency: (fluency by letter/3) + (fluency by category); CVLT: California Verbal Learning Test

Table 1: Demographics and cognitive test scores. memory was assessed using a list-learning task (California Verbal Learning Test, or CVLT), and story recall (Logical Memory of the Wechsler Memory Scale -III) [23]. General cognitive function was assessed using the Modified Mini-Mental State Examination (3MSE) [25]. Raw scores were converted to $\mathrm{z}$-scores using the group mean and standard deviation per test, and z-scores were inverted for Trails-B so that larger scores reflect better performance. The EF composite score was constructed by summing z-scores for Trails-B, Digit-Symbol, Digit Span Backwards, and Fluency, a method used in our previous studies [26]. The verbal memory composite was constructed by summing z-scores for immediate and delayed recall on the CVLT and Logical Memory.

\section{Statistical analysis}

Cognitive composite score outcomes were subjected to separate linear regression models by glucoregulatory marker. Linear regression diagnostics were conducted to test assumptions of linearity, normality, homoscedasticity, and independence of errors, with no violations indicated. Covariates for the regression models included age, education, BMI, and use of antihypertensive medications. Pearson correlations were performed to examine relationships between glucoregulatory markers and performance on individual cognitive tests. We also explored relationships between cognitive and glucoregulatory outcomes and the covariates. Although men were underrepresented in this sample $(n=7)$, the results of t-tests failed to indicate any sex-related differences in outcomes. As a consequence, sex was not included as a covariate in the analyses. Statistical significance was set at $\mathrm{P}<0.05$. Statistical analysis was performed using STATA v11.

\section{Results}

High-normal FPG levels predicted EF $(\beta=-0.41, \mathrm{p}=0.03)$, such that higher FPG concentrations were associated with lower EF composite scores (Figure 1). Similarly, higher FPG levels tended to be associated with poorer verbal memory, although this relationship failed to reach statistical significance $(\beta=-0.34, p=0.06)$ (Figure 2). Neither fasting insulin concentrations nor HbAlc predicted executive function or verbal memory performance (all p-values $>0.05$ ), (data not shown).

Correlational analyses examining relationships between glucoregulatory markers and performance on individual cognitive tests indicated that for the CVLT, higher FPG was associated with poorer immediate $(\mathrm{r}=-0.39, \mathrm{p}=0.02)$ and delayed recall $(\mathrm{r}=-0.38, \mathrm{p}=0.02)$. Similarly, higher FPG predicted worse performance on Digit-Symbol, although this association failed to reach statistical significance $(r=-0.30, p=0.06)$.

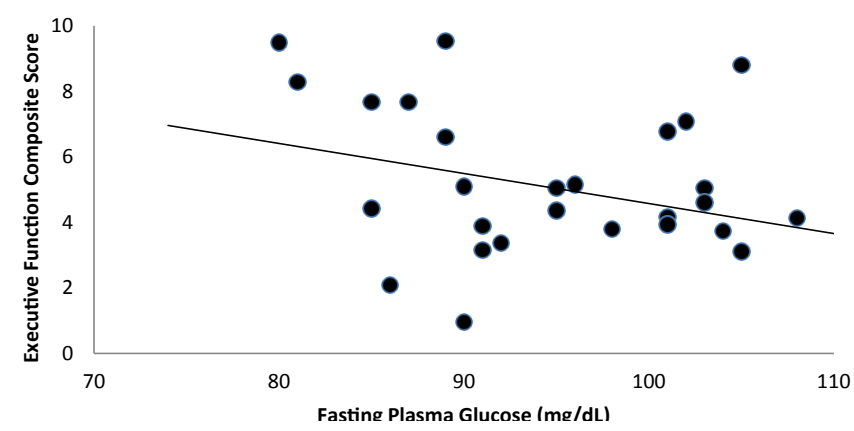

Figure 1: Unadjusted correlation between fasting glucose $(\mathrm{mg} / \mathrm{dL})$ and executive function composite score $(r=-0.35, p=0.05)$. The composite score was constructed by summing z-scores for Trails B of the Trail Making Test, number correct on Digit-Symbol, Digit Span Backwards, and Word Fluency. 


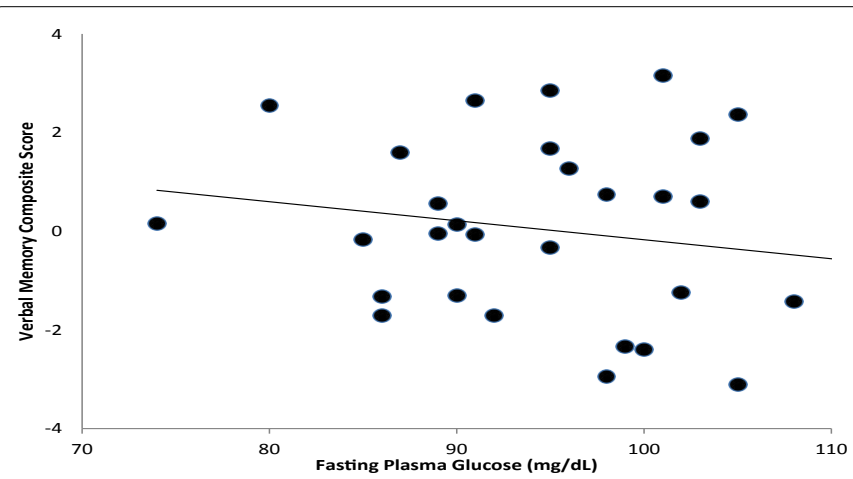

Figure 2: Unadjusted correlation between fasting glucose $(\mathrm{mg} / \mathrm{dL})$ and verbal memory composite score $(r=-0.30, p=0.07)$. The composite score was constructed by summing z-scores for the California Verbal Learning Test (CVLT) and Logical Memory of the Wechsler Memory Scale (WMS-III).

Given that nearly $50 \%$ of the sample was using antihypertensive medications, we explored whether this exposure differentially impacted the primary finding. The results of an ANOVA on EF composite scores compared across groups defined by antihypertensive use (yes vs. no) and FPG classification (normal vs. impaired: $>100 \mathrm{mg} / \mathrm{dL}$ ) indicated a 2 -way interaction $(\mathrm{F}(1,27)=4.91, \mathrm{p}=.03$. Executive function composite scores were lower for adults with impaired FPG, but only among non-users of antihypertensives; among users, executive function was comparable for adults with and without impaired FPG. Although antihypertensive users had higher diastolic $(\mathrm{F}(1,32)=4.81, \mathrm{p}=.03)$ and systolic $(\mathrm{F}(1,32)$ $=4.91, \mathrm{p}=.03)$ blood pressures, when these measurements were included as covariates in the model, the significant 2 -way interaction persisted ( $\mathrm{F}$ $(1,25)=6.04, \mathrm{p}=.02)$. That is, the different impact of FPG on executive function across users and non-users of antihypertensives is not likely attributable to group differences in diastolic or systolic blood pressure.

\section{Discussion}

The current study examined the relationship between markers of glucose/insulin function, as measured by FPG, insulin, and HbAlc, and executive function and verbal memory in African American elders, free of T2DM. Our results extend prior empirical findings from predominately White samples by demonstrating that cognitive abilities most vulnerable to $\mathrm{T} 2 \mathrm{DM}$ and $\mathrm{AD}$ may also be sensitive to variations in FPG even before the threshold of T2DM is crossed $[6,27]$. This relationship was independent of age, education, BMI, and antihypertensive medication use. In the absence of relationships between fasting insulin, $\mathrm{HbAlc}$, and cognition, our preliminary findings suggest that in African Americans without T2DM, FPG may provide unique and important information about cognitive health.

One biological pathway linking peripheral glucose levels to neurocognitive function is neuroendocrine systems. Brain glucose utilization is regionally heterogeneous and prefrontal brain regions are particularly vulnerable to glucose and insulin related metabolic changes $[14,28,29]$. Studies of vascular and structural brain-related changes also link peripheral glucose levels to neurocognitive function [30]. Poor glucose regulation is also associated with increased $\mathrm{AD}$ neuropathology [31]. Unlike FPG, fasting insulin levels and HbA1C were restricted in range, as might be expected in adults without T2DM, which likely accounted for the absence of associations involving these markers in our study. Other studies documenting a relationship between either $\mathrm{HbA1C}$ or fasting insulin and cognition included larger sample sizes and greater variability in insulin and $\mathrm{HbAlc}$ ranges $[32,33]$.
We also report antihypertensive medication use may be protective of EF in older African Americans at increased risk for T2DM. To date, only a few studies have examined associations between antihypertensive use, cognitive function, and glycemic control in older adults [34], therefore it remains unclear as to what effect these medications might have on cognition in adults with prodromal T2DM. Hypertension and T2DM commonly co-occur and both conditions are associated with microvascular and macrovascular disease [35]. Moreover, hypertension is independently associated with executive dysfunction [36]. Additional studies are needed to confirm and further explore this finding, such an interaction involving antihypertensive medication use and executive function in African Americans could have clinically significant implications for clinical care in this cohort.

Limitations of the study include our small sample size, unbalanced gender representation, and cross-sectional design. Also, our estimate of glycemic control was based on a single FPG measurement and therefore may only reflect acute glucoregulatory status. However, our results remain important as FPG is a standard clinical index for screening and management of T2DM, and thus can be a readily accessible tool to identify change in cognitive risk, even before T2DM is diagnosed. Despite these limitations, this study adds to the existing literature. This study examined predictors of cognitive health in a severely understudied cohort of older adults who are at high-risk for T2DM and associated comorbidities, including cognitive impairment. We also utilized robust measures of cognitive function by relying on composite scores that are typically more stable than individual test scores.

In a sample of older African Americans without T2DM, highnormal FPG predicted poorer EF and tended to predict poorer verbal memory, while lower glucose levels were associated with better cognitive performance. Although these findings need to be replicated in larger trials, a critical extension of this work will be to investigate neuropathological correlates of FPG and cognitive function in African Americans without T2DM. These preliminary findings may help inform future trials aimed at prevention strategies to support cognition in prodromal stages of T2DM in minority elders.

\section{Acknowledgments}

Support for this work was provided by grants from the NIA, University of Washington Alzheimer Disease Research Center 2P50AG005136, NIH Training Grant, 5T32 AG000258, and the Geriatric Research, Education and Clinical Center of the Department of Veterans Affairs. Dr. Skinner had full access to all data and takes responsibility for its integrity and the accuracy of the data analysis. The authors thank the staff of the VA Memory Wellness Program and Central Area Senior Center in Seattle, WA.

\section{References}

1. Martín-Timón I, Sevillano-Collantes C, Segura-Galindo A, Del Cañizo-Gómez FJ (2014) Type 2 diabetes and cardiovascular disease: Have all risk factors the same strength? World J Diabetes 5: 444-470.

2. Roberts RO, Knopman DS, Geda YE, Cha RH, Pankratz VS, et al. (2014) Association of diabetes with amnestic and nonamnestic mild cognitive impairment. Alzheimers Dement 10: 18-26.

3. Exalto LG, Whitmer RA, Kappele LJ, Biessels GJ (2012) An update on type 2 diabetes, vascular dementia and Alzheimer's disease. Exp Gerontol 47: 858-864.

4. American Diabetes Assocaition. National Diabetes Statistics Report 2011, Statistics about diabetes.

5. Tang MX, Cross P, Andrews H, Jacobs DM, Small S, et al. (2001) Incidence of $A D$ in African-Americans, Caribbean Hispanics, and Caucasians in northern Manhattan. Neurology 56: 49-56.

6. Messier C, Tsiakas M, Gagnon M, Desrochers A (2010) Effect of age and glucoregulation on cognitive performance. J Clin Exp Neuropsychol 32: 809-821.

7. Ravona-Springer R, Moshier E, Schmeidler J, Godbold J, Akrivos J, et al 
Citation: Skinner JS, Morgan A, Hernandez-Saucedo H, Hansen A, Corbett S (2015) Associations between Markers of Glucose and Insulin Function and Cognitive Function in Healthy African American Elders. J Gerontol Geriat Res 4: 232. doi:10.4172/2167-7182.1000232

Page 4 of 4

(2012) Changes in glycemic control are associated with changes in cognition in non-diabetic elderly. J Alzheimers Dis 30: 299-309.

8. Yaffe K, Blackwell T, Whitmer RA, Krueger K, Barrett Connor E (2006) Glycosylated hemoglobin level and development of mild cognitive impairment or dementia in older women. J Nutr Health Aging 10: 293-5.

9. Osei K, Schuster DP (1994) Ethnic differences in secretion, sensitivity, and hepatic extraction of insulin in black and white Americans. Diabet Med 11: 755-762

10. Nsiah-Kumi P, Ortmeier SR, Brown AE (2009) Disparities in diabetic retinopathy screening and disease for racial and ethnic minority populations--a literature review. J Natl Med Assoc 101: 430-437.

11. Nguyen HT, Grzywacz JG, Arcury TA, Chapman C, Kirk JK, et al. (2010) Linking glycemic control and executive function in rural older adults with diabetes mellitus. J Am Geriatr Soc 58: 1123-1127.

12. Nguyen HT, Arcury TA, Grzywacz JG, Saldana SJ, Ip EH, et al. (2012) The association of mental conditions with blood glucose levels in older adults with diabetes. Aging Ment Health 16: 950-957.

13. Burns CM, Chen K, Kaszniak AW, Lee W, Alexander GE, et al. (2013) Higher serum glucose levels are associated with cerebral hypometabolism in Alzheimer regions. Neurology 80: 1557-1564.

14. Baker LD, Cross DJ, Minoshima S, Belongia D, Watson GS, et al. (2011) Insulin resistance and Alzheimer-like reductions in regional cerebral glucose metabolism for cognitively normal adults with prediabetes or early type 2 diabetes. Arch Neurol 68: 51-57.

15. Cherbuin N, Sachdev P, Anstey KJ (2012) Higher normal fasting plasma glucose is associated with hippocampal atrophy: The PATH Study. Neurology 79: $1019-1026$

16. Weyer C, Hanson RL, Tataranni PA, Bogardus C, Pratley RE (2000) A high fasting plasma insulin concentration predicts type 2 diabetes independent of insulin resistance: Evidence for a pathogenic role of relative hyperinsulinemia. Diabetes 49: 2094-101

17. Okereke OI, Kurth T, Pollak MN, Gaziano JM, Grodstein F (2010) Fasting plasma insulin, C-peptide and cognitive change in older men without diabetes: results from the Physicians' Health Study II. Neuroepidemiology 34: 200-207.

18. Matsuzaki T, Sasaki K, Tanizaki Y, Hata J, Fujimi K, et al. (2010) Insulin resistance is associated with the pathology of Alzheimer disease: the Hisayama study. Neurology 75: 764-770.

19. Farris W, Mansourian S, Chang Y, Lindsley L, Eckman EA, et al. (2003). Insulindegrading enzyme regulates the levels of insulin, amyloid beta-protein, and the beta-amyloid precursor protein intracellular domain in vivo. Proc. Natl Acad Sci 100: $4162-7$

20. Christman AL, Matsushita K, Gottesman RF, Mosley T, Alonso A, et al. (2011) Glycated haemoglobin and cognitive decline: the Atherosclerosis Risk in Communities (ARIC) study. Diabetologia 54: 1645-1652.

21. Gurland BJ, Wilder DE, Lantigua R, Stern Y, Chen J, et al. (1999) Rates of dementia in three ethnoracial groups. Int J Geriatr Psychiatry 14: 481-493.
22. R. Reitan (1958) Validity of the trailmaking test an indicator of organic brain damage. Percept Mot Ski 8: 271-276.

23. D'Amato CP, Denney RL (2008) The diagnostic utility of the Rarely Missed Index of the Wechsler Memory Scale-Third Edition in detecting response bias in an adult male incarcerated setting. Arch Clin Neuropsychol 23: 553-561.

24. Lonie JA, Herrmann LL, Tierney KM, Donaghey C, O'Carroll R, et al. (2009) Lexical and semantic fluency discrepancy scores in aMCl and early Alzheimer's disease. J Neuropsychol 3: 79-92.

25. Teng EL, Chui HC (1987) The Modified Mini-Mental State (3MS) examination J Clin Psychiatry 48: 314-318.

26. Baker LD, Barsness SM, Borson S, Merriam GR, Friedman SD, et al. (2012) Effects of growth hormoneâ€"releasing hormone on cognitive function in adults with mild cognitive impairment and healthy older adults: results of a controlled trial. Arch Neurol 69: 1420-1429.

27. Gluck ME, Ziker C, Schwegler M, Thearle M, Votruba SB, et al. (2013) Impaired glucose regulation is associated with poorer performance on the Stroop Task. Physiol Behav 122: 113-119.

28. Sokoloff L, Reivich M, Kennedy C, Des Rosiers M, Patlax C, et al. (1977) The 14C-deoxyglucose method for measurement of local cerebral glucose utilization: Theory, procedure and normal values in the conscious and anesthetized albino rat J Neurochem 28: 897-916.

29. Craft $S$ (2009) The role of metabolic disorders in Alzheimer disease and vascular dementia: two roads converged. Arch Neurol 66: 300-305.

30. Convit A, Wolf OT, Tarshish C, de Leon MJ (2003) Reduced glucose tolerance is associated with poor memory performance and hippocampal atrophy among normal elderly. Proc Natl Acad Sci U S A 100: 2019-2022.

31. Craft S, Watson GS (2004) Insulin and neurodegenerative disease: shared and specific mechanisms. Lancet Neurol 3: 169-178.

32. van Oijen M, Okereke Ol, Kang JH, Pollak MN, Hu FB, et al. (2008) Fasting insulin levels and cognitive decline in older women without diabetes. Neuroepidemiology 30: 174-179.

33. Peila R, Rodriguez BL, White LR, Launer LJ (2004) Fasting insulin and incident dementia in an elderly population of Japanese-American men. Neurology 63 228-233.

34. Wong MC, Jiang JY, Fung H, Griffiths S, Mercer S (2008) Antihypertensive drug class and impaired fasting glucose: a risk association study among Chinese patients with uncomplicated hypertension. BMC Clin Pharmacol 8: 6.

35. Hassing LB, Hofer SM, Nilsson SE, Berg S, Pedersen NL, et al. (2004) Comorbid type 2 diabetes mellitus and hypertension exacerbates cognitive decline: evidence from a longitudinal study. Age Ageing 33: 355-361.

36. Vicario A, Martinez CD, Baretto D, Diaz Casale A, Nicolosi L (2005) Hypertension and cognitive decline: impact on executive function. J Clin Hypertens (Greenwich) 7: 598-604. 